\title{
Bilateral symmetry of the time course of early facilitation of the Hoffman reflex
}

\author{
GEOFFREY R. HAMMOND and GRAHAM MANN \\ The University of Western Australia, Nedlands, Western Australia, Australia
}

\begin{abstract}
The time course of early facilitation of the Hoffman reflex by a preliminary stimulus that preceded the reflex-eliciting stimulus by $2-17 \mathrm{msec}$ in 1-msec steps was determined separately for the left and right legs of normal male subjects. The time course of recruitment and decay of facilitation was identical for both sides, showing that the asymmetrical temporal processing that has been reported does not extend to a simple neural network in the spinal cord. The results show the early facilitation in more detail than has been reported previously.
\end{abstract}

Clinical evidence that different impairments follow leftsided and right-sided brain damage in humans shows that brain function is asymmetrical but does not show the mechanisms of asymmetrical function. Experimental work on both clinical patients and normal subjects has suggested an asymmetry of temporal resolution with, typically, the left hemisphere able to resolve events in time more finely than the right (see review by Hammond, 1982). The interpretation has been made, in turn, that asymmetrical temporal acuity may contribute to the asymmetry of higher order brain functions (Lackner, 1982; Lackner \& Teuber, 1973; Schwartz \& Tallal, 1980).

The question arises as to whether asymmetrical temporal processing is a property only of complex neural systems (such as those that presumably subserve sensory processing) or whether it is also a property of simple neural networks. Efron's (1963) supposition that different nerve membrane time constants might underlie asymmetrical temporal processing is consistent with the latter.

Simple spinal reflex systems are sensitive to the temporal characteristics of eliciting stimulus conditions and can be used to test whether simple neural systems are asymmetrical in their temporal response properties. The Hoffman reflex (H reflex) is a monosynaptic spinal reflex that can be elicited readily by brief electrical pulses applied to the skin overlying the tibial nerve in the popliteal fossa. A reflex contraction of the soleus muscle follows stimulation after a latency of about $30 \mathrm{msec}$. With constant stimulus and recording conditions, the amplitude of the reflex contraction is a function of the excitability of the alpha motoneuron pool and reflects excitatory and inhibitory neural influences playing on the motoneuron pool. In particular, $\mathrm{H}$-reflex amplitude is sensitive to the interstimulus interval (ISI) between a preliminary conditioning stimulus (S1) and a later reflex-eliciting stimulus (S2). The change in reflex amplitude with ISI describes the recovery function of the reflex. With $\mathrm{S} 1$ below threshold for reflex elicitation, reflex amplitude is augmented above

The authors' mailing address is: Department of Psychology, The University of Western Australia, Nedlands, W.A. 6009, Australia. control levels (established by presentation of S2 alone) at ISIs less than about $10-15$ msec. This early facilitation is followed by a phase of reflex depression at ISIs shorter than about $100 \mathrm{msec}$ that recovers at ISIs of about $200 \mathrm{msec}$ and is followed, in turn, by a later longer lasting depression (Taborikova, 1973; Taborikova \& Sax, 1969).

If the apparent asymmetry of temporal processing in the nervous system is present in simple neural circuits it should be evident in different time courses of recovery functions for the left and right body sides. Goode, Glenn, Manning, and Middleton (1980) reported reliable asymmetrical recovery functions for seven subjects tested. These results do not answer the question posed here because only long ISIs (greater than $30 \mathrm{msec}$ ) were tested. These long ISIs allow the possibility that influences from supraspinal systems (triggered by S1) modulate reflex excitability. As a result, the asymmetries reported by Goode et al. (1980) are not evidence that the spinal mechanisms themselves have asymmetrical temporal properties. We observed changes in H-reflex amplitude over a range of ISIs from 2 to $17 \mathrm{msec}$ (in 1-msec steps) to eliminate S1 effects relayed through supraspinal systems. These observations also extend systematic knowledge of the $\mathrm{H}$ reflex by showing in detail the time course of the early facilitation.

\section{METHOD}

\section{Subjects}

Eleven male subjects, ranging in age from 18 to 44 years (median $=25$ ), were tested. Scores on the Edinburgh Handednes Inventory (Oldfield, 1971) showed three subjects to be left-handed (median decile of left-handedness $=4$ ) and the remainder to be right-handed (median decile of right-handedness $=4.5$ ). These classifications were supported by performance data from a repetitive button-tapping task: all right handers tapped more rapidly with the right hand and two of the three left-handers tapped more rapidly with the left hand. The other left-handed subject was slightly faster with his right hand.

\section{Apparatus and Procedure}

$\mathrm{H}$-reflex recovery functions were determined for both legs in separate runs in each of two sessions that were separated by at least 
3 days. Measurements were taken with the subject seated comfortably in a reclining chair in a quiet, electrically shielded room. The subject was isolated from extraneous noises by white noise delivered over headphones ( $56 \mathrm{dBA}$ ). Recording procedures followed those recommended by Hugon (1973). The foot of the tested leg rested on an inclined plate adjusted so that the angles at the knee and ankle joint were about $120^{\circ}$.

Stimuli were 1-msec square-wave pulses generated by Grass SD9 stimulators and delivered through a silver-plated cathode located over the tibial nerve in the popliteal fossa and a larger gold-plated anode located over the patella. Current levels were regulated by Grass CCU 1 constant-current units.

EMG activity was recorded from the soleus muscle with goldplated electrodes attached to the midline about 2 and $7 \mathrm{~cm}$ superior to the insertion of the muscle on the tendon. A ground electrode was attached over the gastrocnemius muscles between the stimulating and recording electrodes to reduce stimulus artifact. The EMG was amplified by conventional methods using Grass 7P511 amplifiers and was monitored on a storage oscilloscope.

On each run, the threshold for elicitation of the $\mathrm{H}$ reflex was determined by increasing the current of a 1-msec test pulse until an $\mathrm{H}$ reflex was present on two successive stimulations. The intensities of S1 and S2 were set, respectively, at $95 \%$ and $110 \%$ of the threshold value. Pilot work had shown that these intensities produced reliable modulation of reflex amplitude. Seven blocks of trials were run. Each trial block was made up of a control trial (S2 presented alone) and 16 trials in which S1 preceded S2 by one of the ISIs tested $(2,3,4, \ldots, 17 \mathrm{msec})$. The different ISIs were presented randomly with the constraint that all 16 were tested before the next trial block was begun. Intertrial intervals were selected randomly from the set $8,9,10,11$, and $12 \mathrm{sec}$. Stimulus delivery was controlled by microcomputer. Example EMG traces are shown in Figure 1. Analog-to-digital conversion of the amplified EMG was done at $2000 \mathrm{~Hz}$ during a sample period from 20 to $90 \mathrm{msec}$ after S2. Maximum peak-to-peak amplitudes of each reflex within this sample period were computed and stored.

\section{RESULTS}

Figure 2 shows reflex amplitude expressed as a proportion of control amplitude at each ISI for reflexes recorded on the subjects' preferred and nonpreferred sides. The proportions were calculated separately for each block of trials. A systematic change in reflex excitability as a func-

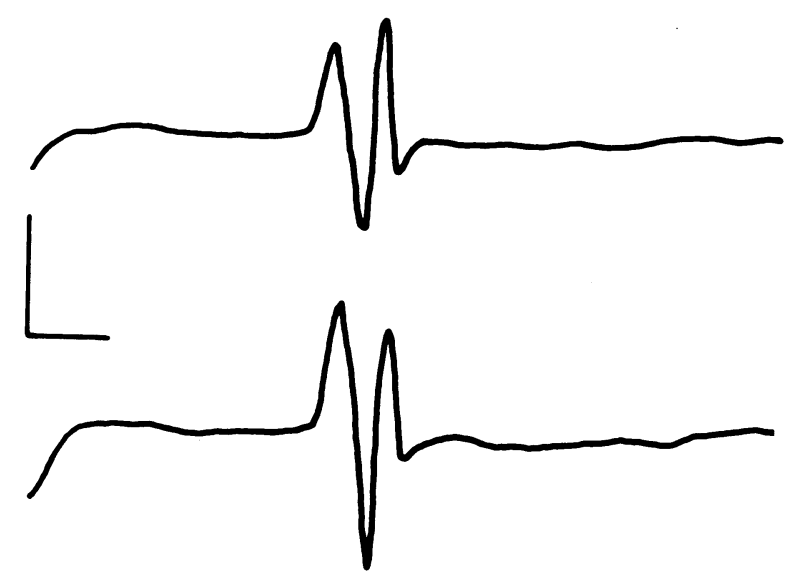

Figure 1. Example tracings of control (above) and facilitated (below) $\mathbf{H}$ reflexes. The vertical and horizontal calibration bars show $500 \mu \mathrm{V}$ and $10 \mathrm{msec}$, respectively.

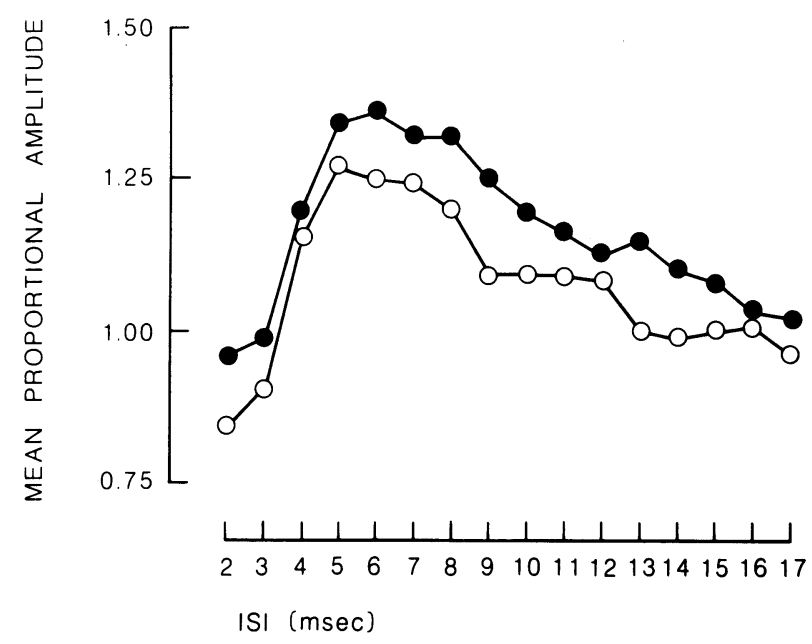

Figure 2. Mean proportional reflex amplitude as a function of interstimulus interval on the preferred (closed circles) and nonpreferred (open circles) sides.

tion of ISI is evident, with some inhibition at the shortest ISIs followed by a rapidly developing facilitation that peaked at 5-6 msec and thereafter decayed more slowly back to control levels. Analysis of variance showed a reliable main effect of ISI $[F(15,150)=13.66, p<.05]$. Dunnett's $t$ tests showed that the inhibition of reflex excitability at the 2-msec ISI was not reliable and that there was reliable facilitation at the 5-, 6-, 7-, and 8-msec ISIs.

The difference in excitability level between reflexes elicited on the subjects' preferred and nonpreferred sides was not reliable $(F<1)$. The slopes of the best-fitting lines of recruitment and decay of reflex facilitation (taken as the changes in proportional reflex amplitude from 2-6 msec and from 6-17 msec, respectively) were the same for both sides, 0.12 for the recruitment limb and -0.03 for the decay limb. No result was changed by casting the results according to "left" and "right" instead of "preferred" and "nonpreferred" or by analyzing results from left-handed and right-handed subjects separately.

\section{DISCUSSION}

The present results show that there is no asymmetry in the time course of either recruitment or decay of the early facilitation of the $\mathrm{H}$ reflex when the reflex-eliciting stimulus is preceded by a preliminary conditioning stimulus in the range from 2 to $17 \mathrm{msec}$. The temporal parameters of change in alpha motoneuron excitability are the same on both sides of the spinal cord. The implication of this finding is that the asymmetries in temporal acuity that have been reported emerge from the organization of higher order systems of neurons and are not primitive properties of individual neurons or simple neural circuits.

The results complement other observations of the $\mathrm{H}$ - 
reflex recovery function by showing the changes in excitability at brief ISIs in detail. In particular, a preliminary stimulus that is below threshold for reflex elicitation produces a rapidly growing facilitation that peaks about 5-6 msec and then declines more slowly back to control levels. This early phase of reflex facilitation has not been described as fully in previous work that has noted its existence (Magladery, Teasdall, Park, \& Porter, 1951; Zander Olsen \& Diamantopoulos, 1967). Lloyd (1946) showed that the facilitation obtained by delivery of $S 1$ and S2 to separate branches of the afferent nerves of monosynaptic reflexes was maximal when the stimuli were simultaneous and decayed exponentially, reaching control levels at about 10-12 msec. This facilitation has been attributed to an S1-produced subliminal fringe: motoneurons that are excited subliminally for a brief period of time, resulting in greater motoneuron discharge to $S 2$. The changes in reflex excitability described here are probably a composite of this subliminal-fringe-produced facilitation and inhibition that results from refractory effects in the nerve fibers at the 2-3-msec ISIs (see, e.g., Brinley, 1974, for a discussion of refractory effects in nerve fibers).

Excitation of a subliminal fringe of motoneurons by a preliminary stimulus increases the amplitude of a subsequently elicited reflex but leaves its latency unchanged. The amount of amplitude facilitation depends upon the size of the subliminal fringe, which, in turn, depends upon the intensity of the preliminary stimulus (Patton, 1965). Most studies of suprasegmental reflexes (such as acoustic startle) have found that preliminary stimuli presented at brief ISIs reduced reflex latency without a corresponding change in amplitude (Hoffman \& Ison, 1980). However, strong amplitude facilitation, together with latency reduction, has been observed with acoustic startle (Ison, McAdam, \& Hammond, 1973). This difference in outcome may be attributable to the intensity of the preliminary stimulus: relatively weak preliminary stimuli may facilitate reflex latency without affecting reflex amplitude, whereas more intense preliminary stimuli (such as those used by Ison et al., 1973) may facilitate both reflex latency and amplitude. This conjecture is consistent with attribution of early facilitation of amplitude to a subliminal fringe that is dependent on the intensity of the preliminary stimulus.

\section{REFERENCES}

BRINLEY, F. J. (1974). Excitation and conduction in nerve fibers. In V. B. Mountcastle (Ed.), Medical physiology (13th ed., Vol. 1, pp. 34-76). Saint Louis: Mosby.

EFron, R. (1963). Temporal perception, aphasia and deja vu. Brain, 86, 403-424.

Goode, D. J., Glenn, S., Manning, A. A., \& Middleton, J. F. (1980). Lateral asymmetry of the Hoffman reflex: Relation to cortical laterality. Journal of Neurology, Neurosurgery and Psychiatry, 43, 831-835.

Hammond, G. R. (1982). Hemispheric differences in temporal resolution. Brain \& Cognition, 1, 95-118.

Hoffman, H. S., \& Ison, J. R. (1980). Reflex modification in the domain of startle: I. Some empirical findings and their implications for how the nervous system processes sensory input. Psychological Review, 87, 175-189.

Hugon, M. (1973). Methodology of the Hoffman reflex in man. In J. E. Desmedt (Ed.), New developments in electromyography and clinical neurophysiology. Vol. 3: Human reflexes, pathophysiology of motor systems, methodology of human reflexes (pp. 271-293). Basel: Karger.

Ison, J. R., McAdam, D. W., \& Hammond, G. R. (1973). Latency and amplitude changes in the acoustic startle reflex of the rat produced by variation in auditory prestimulation. Physiology \& Behavior, 10, 1035-1039.

LACKNER, J. R. (1982). Alterations in resolution of temporal order after cerebral injury in man. Experimental Neurology, 75, 501-509.

Lackner, J. R., \& Teuber, H.-L. (1973). Alterations in auditory fusion thresholds after cerebral injury in man. Neuropsychologia, 11, 409-415.

LLOYD, D. P. C. (1946). Facilitation and inhibition of spinal motoneurons. Journal of Neurophysiology, 9, 421-438.

Magladery, J. W., Teasdall, R. D., Park, A. M., \& Porter, W. E. (1951). Electrophysiological studies of nerve and reflex activity in normal man. V. Excitation and inhibition of two-neurone reflexes by afferent impulses in the same nerve trunk. Bulletin of The Johns Hopkins Hospital, 88, 520-537.

OLDFIELD, R. C. (1971). The assessment and analysis of handedness: The Edinburgh Inventory. Neuropsychologia, 9, 97-113.

Patton, H. D. (1965). Spinal reflexes and synaptic transmission. In T. C. Ruch, H. D. Patton, J. W. Woodbury, \& A. L. Towe (Eds.), Neurophysiology (2nd ed., pp. 153-180). Philadelphia: Saunders.

SChwartz, J., \& TAllal, P. (1980). Rate of acoustic change may underlie hemispheric specialization for speech perception. Science, 207, 1380-1381.

TABORIKova, H. (1973). Supraspinal influences on H-reflexes. In J. E. Desmedt (Ed.), New developments in electromyography and clinical neurophysiology. Vol. 3: Human reflexes, pathophysiology of motor systems, methodology of human reflexes ( $\mathrm{pp}$. 328-335). Basel: Karger.

TABorikova, H., \& SAX, D. S. (1969). Conditioning of H-reflexes by a preceding subthreshold H-reflex stimulus. Brain, 92, 203-212.

Zander Olsen, P., \& Diamantopoulos, E. (1967). Excitability of spinal motor neurones in normal subjects and patients with spasticity, Parkinsonian rigidity, and cerebellar hypotonia. Journal of Neurology, Neurosurgery, and Psychiatry, 30, 325-331. 